\title{
Development of a Spatial Domain Decomposition Scheme for Monte Carlo Neutron Transport
}

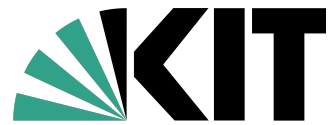

Karlsruher Institut für Technologie

\section{García ${ }^{1}$, D. Ferraro ${ }^{1}$, L. Mercatali ${ }^{1}$, V. Sanchez ${ }^{1}$, J. Leppänen ${ }^{2}$, V. Valtavirta ${ }^{2}$}

26th ICONE - International Conference on Nuclear Engineering London, UK, July 22-26, 2018

${ }^{1}$ Karlsruhe Institute of Technology (KIT), ${ }^{2}$ Technical Research Centre of Finland (VTT) 


\section{Motivation}

- McSAFE project:

- Full-core pin-by-pin LWR analysis based on the Monte Carlo method.

- Steady-state, depletion and transient problems.

- High performance Monte Carlo neutron transport (Serpent2).

- Multiphysics: thermalhydraulics and fuel performance feedback. 


\section{Motivation}

- McSAFE project:

- Full-core pin-by-pin LWR analysis based on the Monte Carlo method.

- Steady-state, depletion and transient problems.

- High performance Monte Carlo neutron transport (Serpent2).

- Multiphysics: thermalhydraulics and fuel performance feedback.

- Main challenges for Monte Carlo codes:

- Efficient variant reduction for steady-state and transient problems.

- Massive parallelization with MPI-OpenMP (speedup optimization).

- Huge memory demand (memory footprint reduction or distribution). 


\section{Motivation}

- McSAFE project:

- Full-core pin-by-pin LWR analysis based on the Monte Carlo method.

- Steady-state, depletion and transient problems.

- High performance Monte Carlo neutron transport (Serpent2).

- Multiphysics: thermalhydraulics and fuel performance feedback.

- Main challenges for Monte Carlo codes:

- Efficient variant reduction for steady-state and transient problems.

- Massive parallelization with MPI-OpenMP (speedup optimization).

- Huge memory demand (memory footprint reduction or distribution).

- Parallel schemes for Monte Carlo particle transport:

- Particle-based parallelism.

- Data decomposition.

- Spatial domain decomposition. 


\section{Motivation}

- McSAFE project:

- Full-core pin-by-pin LWR analysis based on the Monte Carlo method.

- Steady-state, depletion and transient problems.

- High performance Monte Carlo neutron transport (Serpent2).

- Multiphysics: thermalhydraulics and fuel performance feedback.

- Main challenges for Monte Carlo codes:

- Efficient variant reduction for steady-state and transient problems.

- Massive parallelization with MPI-OpenMP (speedup optimization).

- Huge memory demand (memory footprint reduction or distribution).

- Parallel schemes for Monte Carlo particle transport:

- Particle-based parallelism.

- Data decomposition.

- Spatial domain decomposition. 


\section{Introduction}

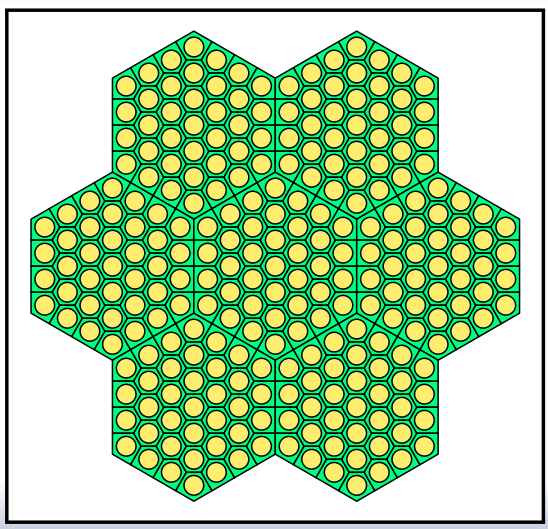

M. Garcia - A SDD Scheme for Monte Carlo Transport - ICONE26, London, UK 


\section{Introduction}

\section{Overview of SDD}

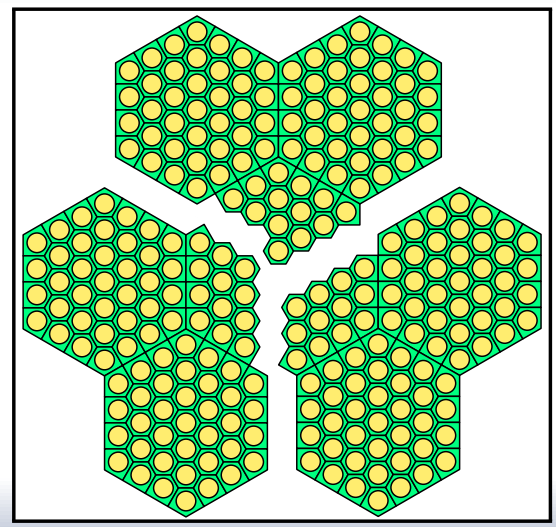

M. Garcia - A SDD Scheme for Monte Carlo Transport - ICONE26, London, UK 


\section{Introduction}

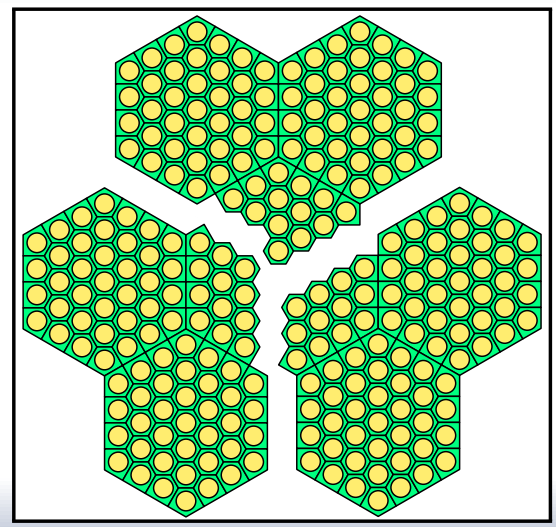

- Key issues:

- Geometry partition.

- Fission source scaling.

- Particle domain crossings.

- Tracking loop termination.

- Gathering of results. 


\section{Overview of SDD}

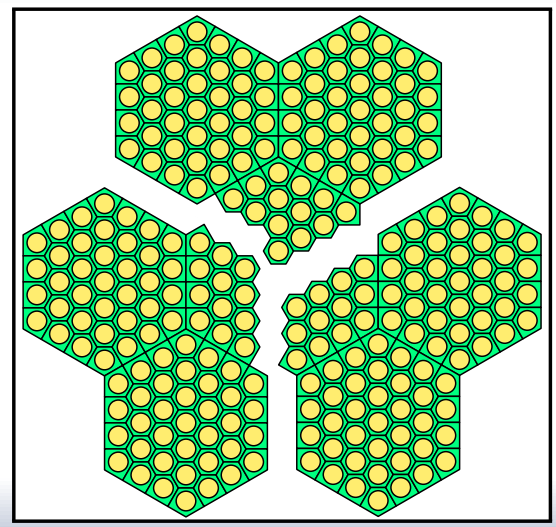

- Key issues:

- Geometry partition.

- Fission source scaling.

- Particle domain crossings.

- Tracking loop termination.

- Gathering of results.

- Potential benefits:

- Distribution of the memory demand across nodes (MPI).

- Data locality (nuclear properties, material compositions, tallies).

- Very good speedup. 


\section{Overview of SDD}

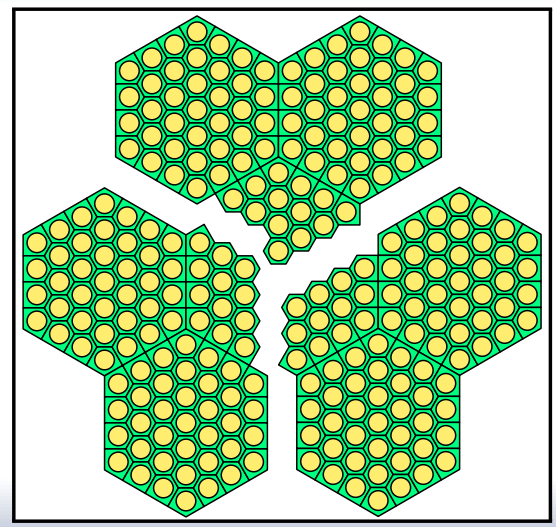

- Key issues:

- Geometry partition.

- Fission source scaling.

- Particle domain crossings.

- Tracking loop termination.

- Gathering of results.

- Potential benefits:

- Distribution of the memory demand across nodes (MPI).

- Data locality (nuclear properties, material compositions, tallies).

- Very good speedup. 


\section{Domain crossing}

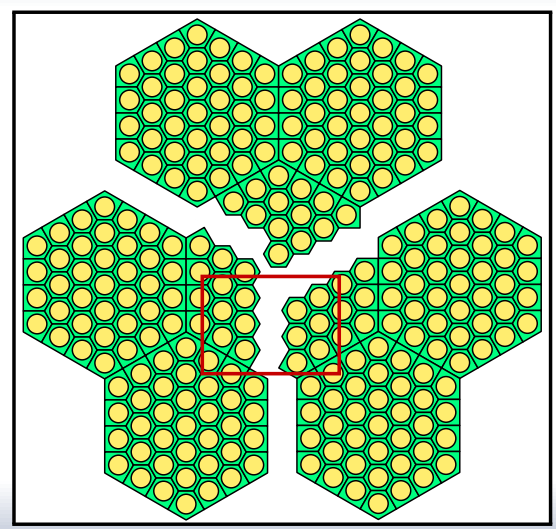

M. Garcia - A SDD Scheme for Monte Carlo Transport - ICONE26, London, UK 


\section{Domain crossing}
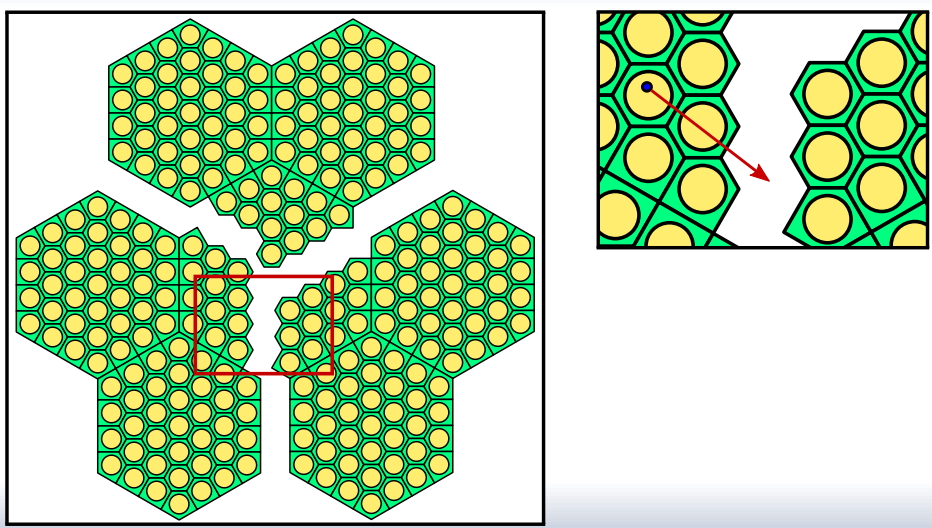

M. Garcia - A SDD Scheme for Monte Carlo Transport - ICONE26, London, UK 


\section{Domain crossing}
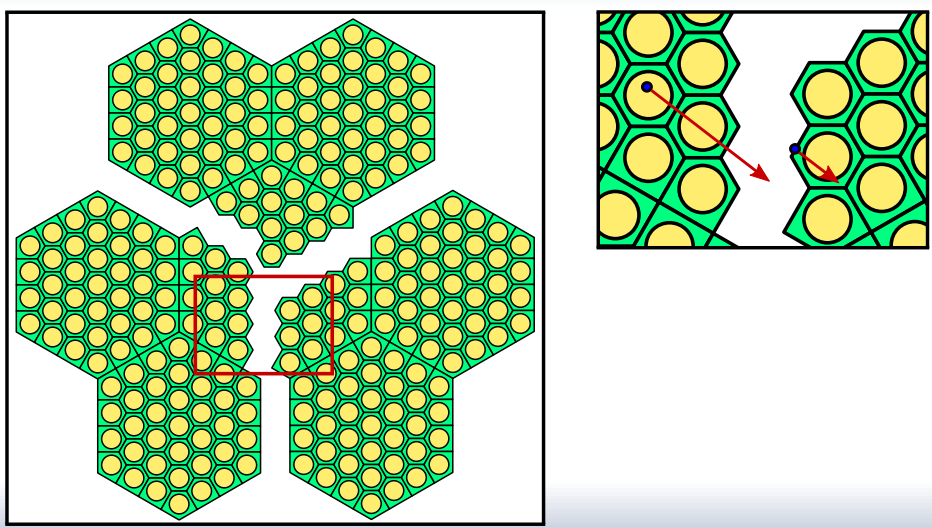

M. Garcia - A SDD Scheme for Monte Carlo Transport - ICONE26, London, UK 


\section{Domain crossing}
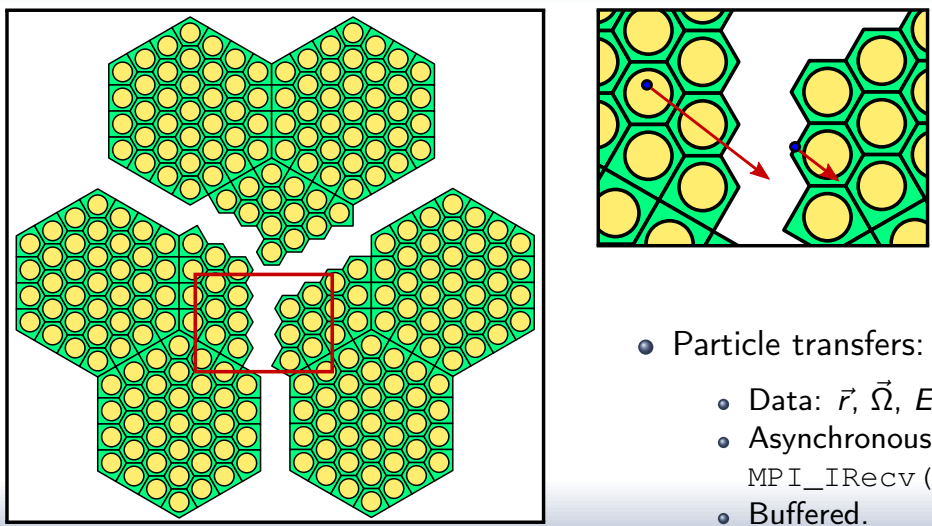

- Particle transfers:

- Data: $\vec{r}, \vec{\Omega}, E, t, w$, etc.

- Asynchronous (MPI_ISend (), MPI_IRecv ()).

- Buffered. 


\section{Tracking termination}

- Termination condition:

- All local histories have to be completed.

- All sent particles have to be recieved.

- Global operation.

- Not trivial due to asynchronous particle communications. 


\section{Tracking termination}

- Termination condition:

- All local histories have to be completed.

- All sent particles have to be recieved.

- Global operation.

- Not trivial due to asynchronous particle communications.

- Synchronization:

- A correct particle balance is obtained.

- As few as posible for performance. 


\section{Tracking termination}

- Termination condition:

- All local histories have to be completed.

- All sent particles have to be recieved.

- Global operation.

- Not trivial due to asynchronous particle communications.

- Synchronization:

- A correct particle balance is obtained.

- As few as posible for performance.

- Asynchronous estimation:

- The particle balance can be estimated without synchronization.

- Synchronization can be requested when this estimation matches. 


\section{Implementation}

\section{Particle tracking loop}

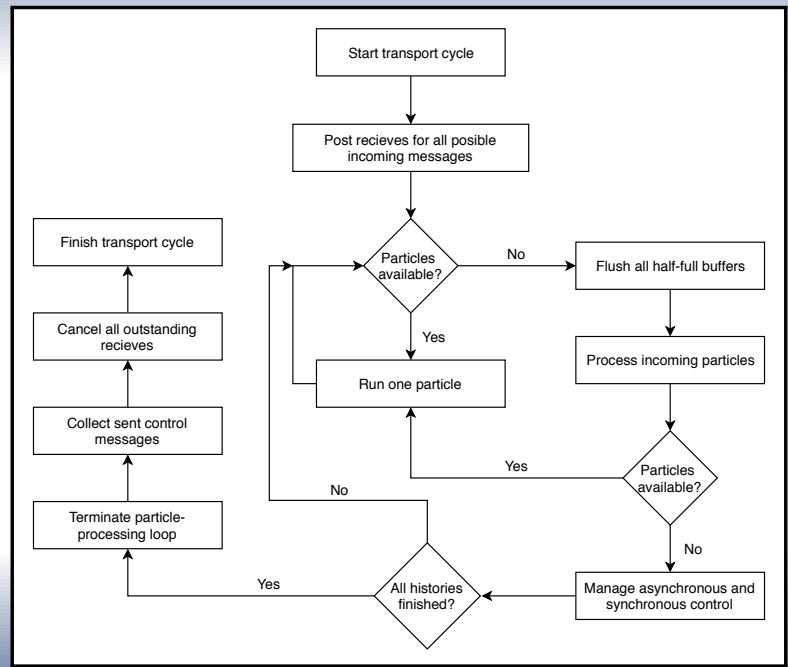

M. Garcia - A SDD Scheme for Monte Carlo Transport - ICONE26, London, UK 


\section{Test program}

- No transport.

- Neutrons born in a domain escape with probability $p_{e}$.

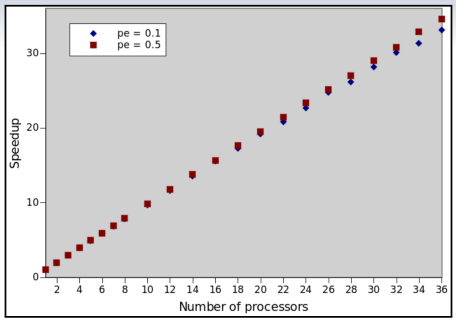

- Uniform source.

- Average tracking time taken from Serpent2.

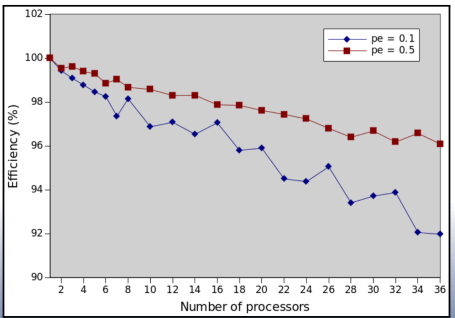

M. Garcia - A SDD Scheme for Monte Carlo Transport - ICONE26, London, UK 


\section{Serpent2}

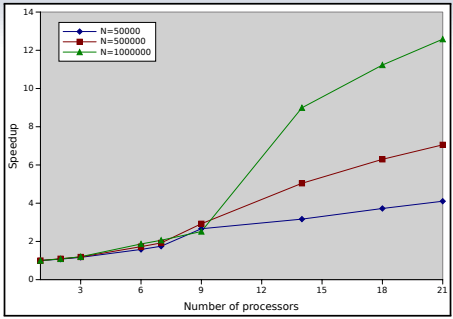

- VVER-440 pin-by-pin fuel assembly.

- Pure MPI (no OpenMP).

- Simplified algorithm.
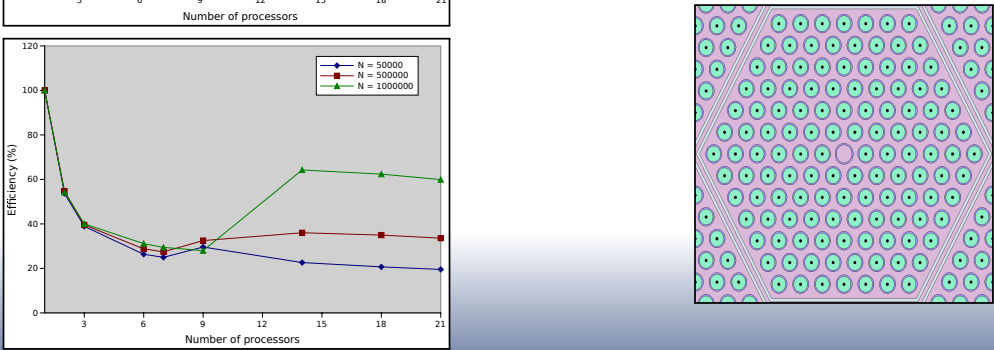

M. Garcia - A SDD Scheme for Monte Carlo Transport - ICONE26, London, UK 


\section{Current status and future work}

- Current status:

- SDD communications scheme implemented, tested and optimized.

- Implementation in Serpent2 underway.

- Geometry partition being developed.

- Future work:

- Development of an MPI-OpenMP optimized algorithm.

- Further optimization and verification.

- McSAFE project:

- Potential capabilities for pin-by-pin full-core simulation.

- Optimization of parallel multiphysics schemes. 


\section{Questions?}

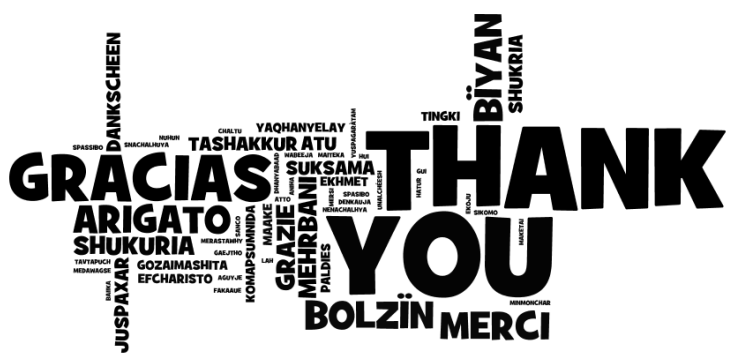


Appendices 


\section{Particle communications}
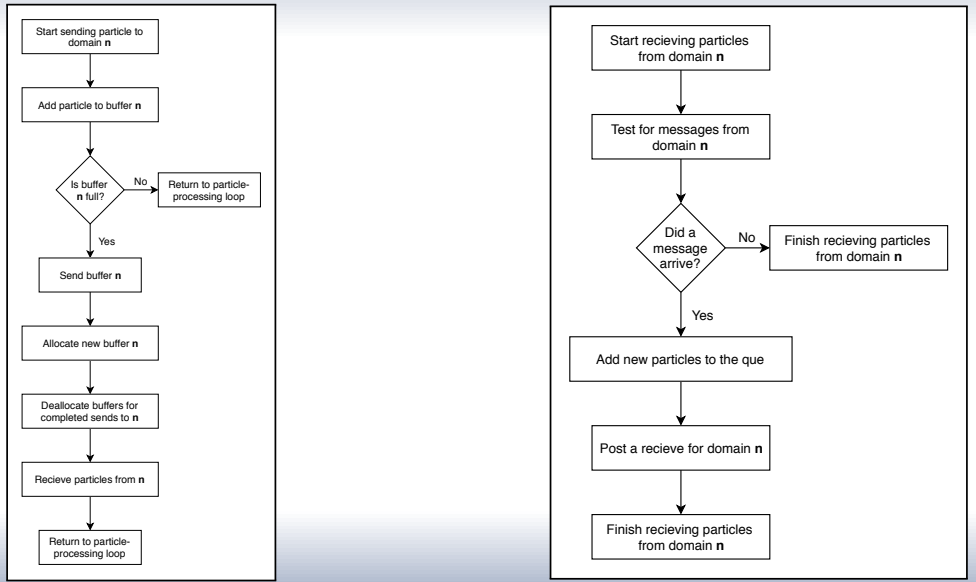

M. Garcia - A SDD Scheme for Monte Carlo Transport - ICONE26, London, UK 


\section{Tracking termination}

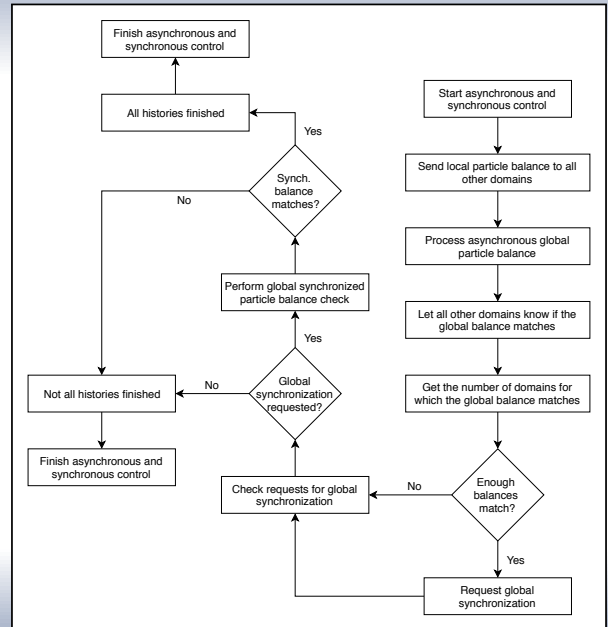

M. Garcia - A SDD Scheme for Monte Carlo Transport - ICONE26, London, UK 